\title{
TAURUS OBSERVATIONS OF SO POLAR RING GALAXIES
}

\author{
R.A. Nicholson ${ }^{1}$, K. Taylor ${ }^{1}$, W.B. Sparks ${ }^{1} \&$ J. Bland ${ }^{1,2}$ \\ ${ }^{1}$ Royal Greenwich Observatory, Hailsham, Sussex, England \\ 2 Inst. for Astron., Univ. of Hawaii, Honolulu, USA
}

By use ot the TAURUS imaging Fabry-Perot interferometer (Taylor \& Atherton 1980) we have obtained seeing limited two-dimensional velocity, line width and line flux maps of the ionised gas in two polar ring systems.

The H $\alpha$ TAURUS maps of NGC $4650 A$, obtained at the AAT, show clearly an extended $\left(110^{\prime \prime}\right)$ differentially rotating gas disc orthogonal to the projected minor axis of the galaxy. The gas warps in a manner consistent with it being in a polar orbit about an S0 galaxy, and stabilised by its self gravity (Sparke 1986).

NGC 2685 was observed with the 2.5-m Isaac Newton Telescope at the Observatory del Roque de Los Muchachos, La Palma. This has revealed the distribution of HII regions surrounding the stellar component. Provisional results show no sign of the complicated central warp structure seen in NGC 4650A. Evidence is found for possible severe warping at radii greater than $4 \mathrm{kpc}\left(\mathrm{H}_{0}=50 \mathrm{~km} / \mathrm{s} / \mathrm{Mpc}\right)$.

\section{OBSERVATIONS/RESULTS}

Figure 1 shows the velocity contour map obtained from the TAURUS data cube on NGC 4650A. The velocity field is found to be far more complex in structure than that expected from a laminar disc undergoing differential rotation. Figures $2 a, b$ show the line of nodes and rotation curve obtained by sampling this velocity field with a series of concentric inclined circular annuli. The rotation and line of node curves were then used in modelling the velocity field expected from a thin warped disc. The resultant model reproduces well the double kinked ' $M$ ' type perturbations seen in the central regions of the contoured data map, Figure 3. Lausten and West (1980) suggested that the observed intensity distribution might be the result of the disc warping across our line of sight: this is supported by the thin disc model.

Preliminary analysis of NGC 2685 reveals a gas disc inclined at 15 degrees to the minor axis of the stellar system. Evidence for warping in the outer regions of this disc, and HII regions associated with the outer equatorial HI ring (Shane 1980 ) is found. The existence of two nearly orthogonal gas geometries may be explanable in terms of a severe ( $\sim 90 \mathrm{deg}$ ) warp. Such a warp would give a natural explanation to the peculiar asymmetric distribution of dust bands seen across the face of the stellar component. 
Fig 1

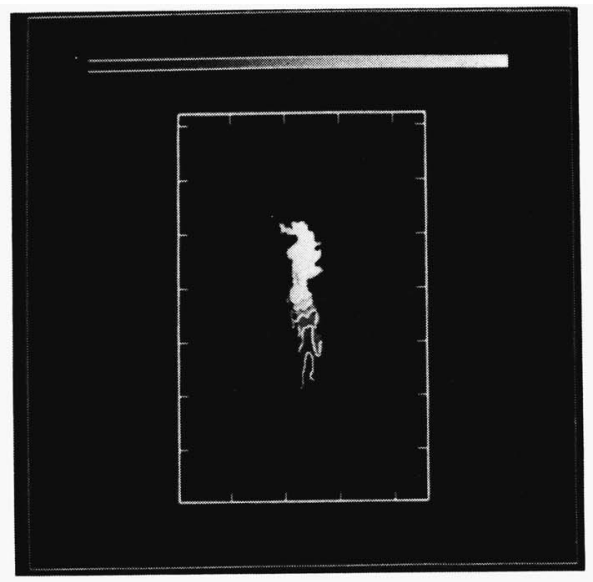

Fig 2b
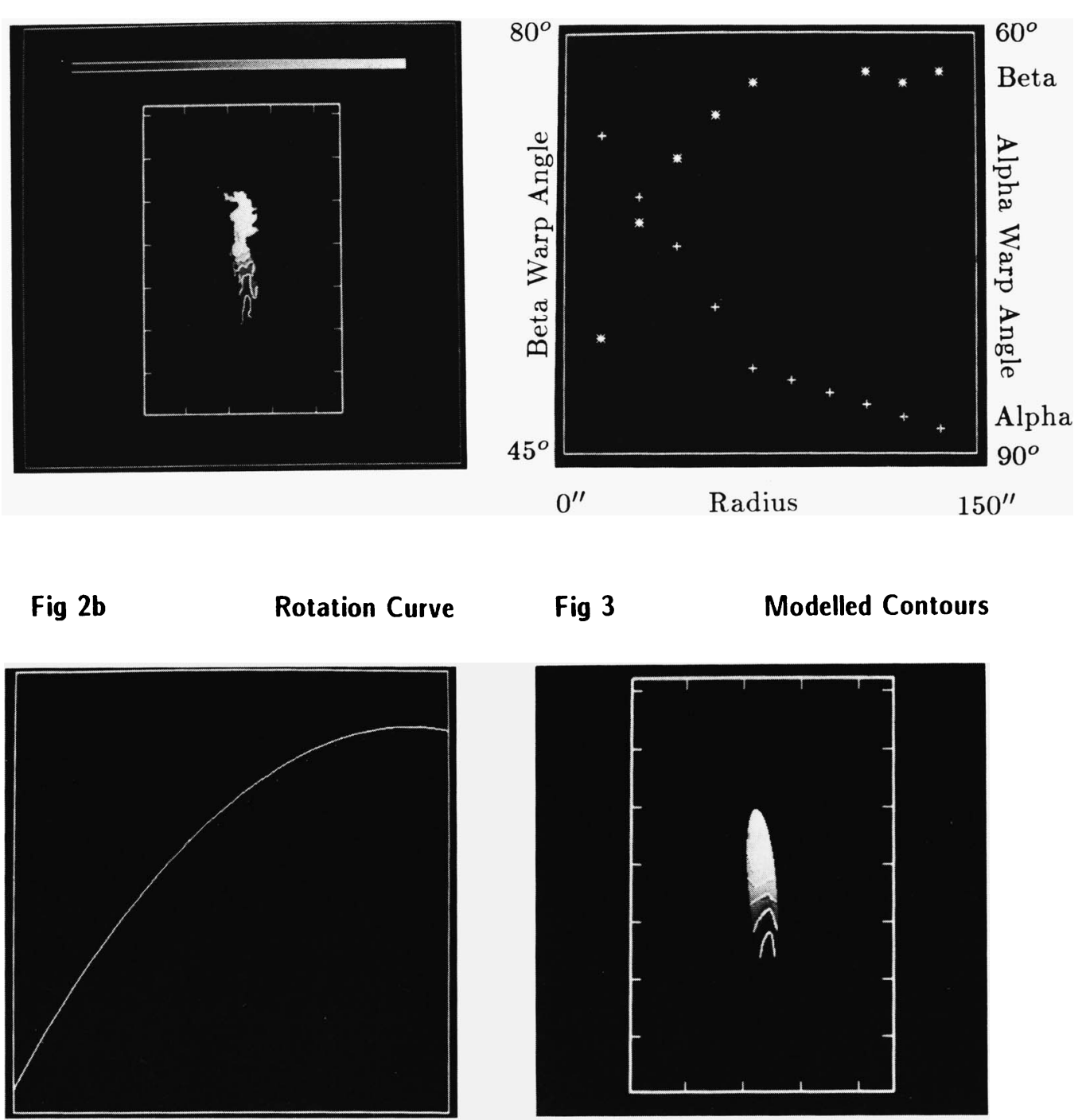

Fig 3

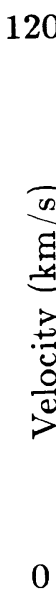

$0^{\prime \prime}$
Rotation Curve

Radius
Fig 2a

Alpha/Beta Warps

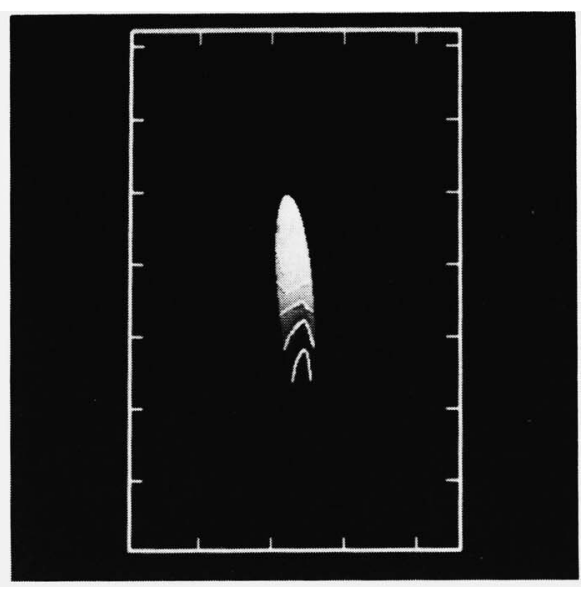

\section{REFERENCES}

Lausten, S., \& West, R.M., 1980. Journ. Astrophys. Astron., 1, 177.

Shane, W.W., 1980. Astr. Astrophys., 82, 314.

Sparke, L.S., 1986. Mon. Not. R. astr. Soc., 219, 657.

Taylor, K., \& Atherton, P.D., 1980. Mon. Not. R. astr. Soc., 191, 675. 